\title{
Axisymmetric elasto-plastic flexure of circular plates in the large deflexion regime
}

\author{
G. J. TURVEY
}

\section{Dr T. K. Chaplin, University of Birmingham}

The Author's use of Day's original dynamic relaxation method to solve the large deflexion equations for a circular plate would be relevant to plates used in various fields. For example, after large foundation settlements a tank base plate would almost certainly enter the plastic region.

28. I would like to see some comparison of the efficiency of dynamic relaxation, expressed in computer time, core storage needed and total program statements, with corresponding measures for one or more other methods such as direct Gaussian elimination or the successive over-relaxation iterative method. Such a comparison (or information to make future comparisons possible) would help in the choice of the most suitable method for similar problems.

29. Dynamic relaxation is a second-order (accelerated) method for solving suitable equations derived in any way, e.g. from finite elements, not only those from finite difference approximations. The equations are handled as if the variables represent nodal forces and motions. Position adjustments are made simultaneously at every node, as in the accelerated Jacobi method which dynamic relaxation so closely resembles. Every node has a viscous damper for each independent direction of motion, controlled only by the corresponding velocity at that one node. The damping constant for each direction of motion would typically be chosen to give substantial under-damping of its fundamental mode, and might well be the same for every node. Higher modes are therefore severely over-damped, and hence they decay far too slowly. When the motions have effectively ceased, the deflexions give the solution of the equations.

30. What was the criterion of solution accuracy chosen by the Author, how was it applied and how many cycles of iteration were needed to reach it in the worst case? What were the increments of load, and was the full criterion of accuracy applied after every increment or just the last one? How was the damping determined, and what proportion of critical damping was it? Could the Author show for a few nodes the difference between final deflexion and current deflexion, plotted against iteration number?

31. Some years ago I discovered an explicit general rule for finding near-optimal damping constants for dynamic relaxation to help a user with no previous knowledge of a particular problem. Automatic design was also envisaged, in which parameters are changed (smoothly or in steps) during the run. The clue came from Fourier series and the bending of a simply supported beam without ground support.

Paper published: Proc. Insin Civ. Engrs, Part 2, 1979, 67, Mar., 81-92. 
32. The load per unit length on the beam is

$$
q=E I \partial^{4} w / \partial x^{4}
$$

where $E I$ is flexural rigidity, $w$ is deflexion and $x$ is distance. The $n$th term in the Fourier series ( $n$th mode of sinusoidal deflexion) is

$$
w_{n}=A \sin (n \pi x / L)
$$

where $L$ is the length. Combining equations (6) and (7) gives

$$
\begin{aligned}
q_{n} & =A E I\left(n^{4} \pi^{4} / L^{4}\right) \sin (n \pi x / L) \\
& =E I\left(n^{4} \pi^{4} / L^{4}\right) w_{n} .
\end{aligned}
$$

As $q$ is exactly proportional to $w$ at every point on the beam in equation (8), each Fourier term is an eigenfunction.

33. From this it was realized that by detecting the apparent form of current deformation from motions at surrounding nodes, the damping factor could be automatically calculated and made to change smoothly as the computation proceeds. In reference 22 it is shown that there are particularly simple explicit damping expressions, based on rate of change of curvature, for small deflexion bending of beams and plates (both without ground support), and expressions for handling non-linear equations (e.g. for large deflexion theory of plate bending) are given.

34. The term 'self-adaptive iteration' might be used for such a method, where the acceleration constant (or its analogue, such as damping in dynamic relaxation) is changed automatically on a rational basis during the run. In Southwell's hand relaxation procedure, the optimal strategy was usually said to be initial broad block changes followed by line relaxation, with point relaxation left until near the end. The nearoptimal strategy for self-adaptive iteration on a digital computer seems to be just the opposite. At first the damping is large and acceleration small, to smooth out the short wavelength errors; towards the end the damping is usually rather small, to apply critical damping to the longest wavelength errors (lowest natural frequency mode) and thereby give the maximum acceleration. Except where the loads in a linear problem happen to be an eigenvector, in general the damping is different at every node, even for one direction of motion.

35. Self-adaptive iteration is also intended to give the smoothest possible approach (consistent with speed) of every nodal deflexion towards its final value. Where continuing smoothness of change is not of prime importance, some improvement in speed is possible by the judicious use of under-damping in combination with other techniques.

36. For a bending problem, the damping constant might well be made almost irrelevant with suitable handling; even zero damping has worked quite well in a few simple trials.

\section{Dr Turvey}

In $\$ 28$ Dr Chaplin expresses a wish to see an efficiency comparison, in terms of computer run time, core storage and total program statements, made between dynamic relaxation and other methods such as Gaussian elimination and successive overrelaxation. I have not made a comparison of this type and so I can only suggest that some qualitative guidance (probably only applicable to linear problems) may be drawn from the work of Otter, ${ }^{23.24}$ Wood $^{25}$ and Hodgkins. ${ }^{26}$ However, I believe that the choice of method of solution should not rest solely on the criteria posed by Dr Chaplin. Although dynamic relaxation may be computationally slower than successive overrelaxation it does have the attribute that the computations resemble (as indicated in $\$ 29$ ) the physical, damped vibrating system. Thus, not only is physical insight maintained, but regular monitoring of the calculations during the computing process enables the solution convergence to be appreciated in a physically meaningful fashion. Further, the dynamic relaxation algorithm is simple and readily programmed (these features may or may not be characteristic of other solution methods). From an engineer's 
standpoint, I believe that these attributes render the dynamic relaxation method an attractive tool for solving complex structural and other problems.

38. In $\S 30 \mathrm{Dr}$ Chaplin raises a number of queries about solution accuracy, numbers of load increments and the selection of the damping factors. There are a number of ways in which the adequacy of solution convergence may be decided on, e.g. satisfactory convergence may be presumed when the velocities, the kinetic energies or the equilibrium equation residuals are all less than a specified small quantity. In applying the dynamic relaxation method to non-linear structural problems I have usually opted, as in the present instance, to monitor velocities to establish convergence. Experience with the present problem showed that, in the worst cases, velocities were acceptably small (absolute value $<10^{-6}$ ) after 700 iterations, although in many instances 200-250 iterations sufficed.

39. In its present form, the computer program is unable to cope in a general manner with elastic unloading-the necessary coding has not yet been incorporated. Hence, the load range for each plate aspect ratio and support condition was selected such that substantial unloading did not occur (this was readily established as applied load magnitudes consistent with substantial elastic unloading led to failure to converge and/or computational instability). It was, therefore, not necessary to operate the computer program in a truly incremental fashion. Indeed, loads were selected arbitrarily within the load range applicable to each of the problems under consideration and a separate computer run with full convergence was undertaken for each. Thus, a minimum of five and a maximum of thirteen load values were used in establishing the load-deflexion curves. It is recognized that this approach may be regarded as inefficient. However, it overcomes the difficulty associated with having to adjust the damping factors automatically as the load on the plate increases. If the computations are performed in a truly incremental manner, then an a priori knowledge of the load-damping factor relationship is required if wasted (i.e. unconverged) computer runs are to be avoided.

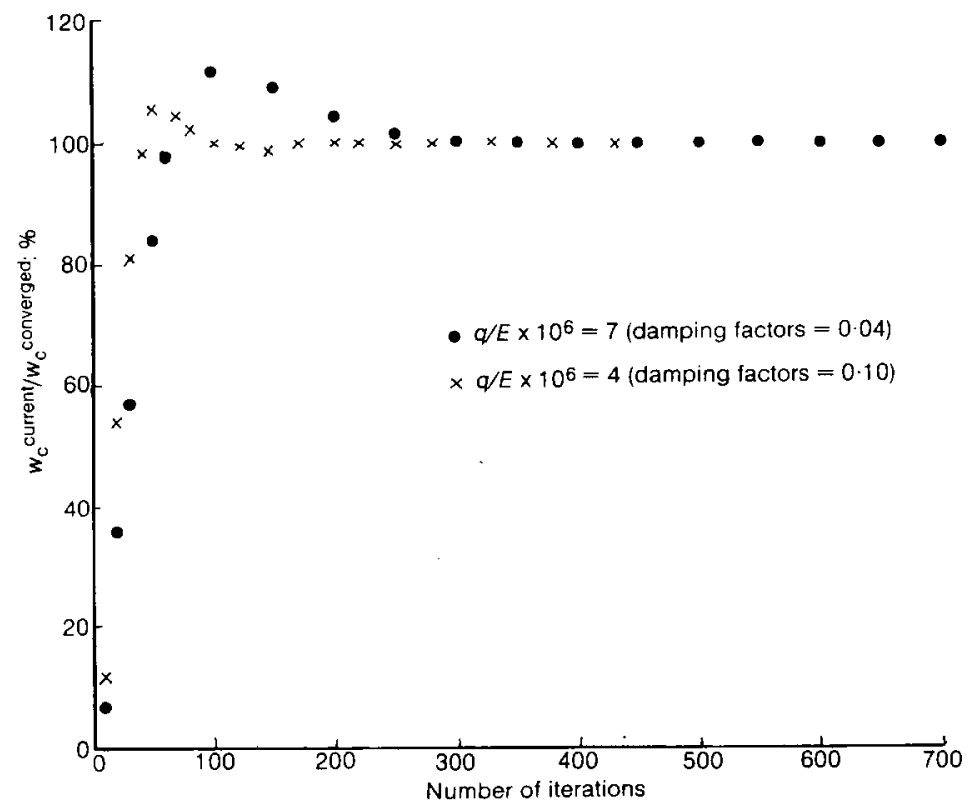

Fig. 7. Clamped circular plate $\left(r_{0} / h=10, s / E=1 \cdot 25\right)$ 


\section{DISCUSSION}

The approach I adopted minimizes this waste, especially as $I$ rely on experience with this type of problem in deciding on damping factor values. No attempt has been made to employ a more rigorous approach, such as using a certain percentage of critical damping, in selecting damping factors. Indeed, I doubt whether it is more rigorous where non-linear problems are concerned and, in any case, the calculation of critical damping is itself not always simple.

40. Figure 7 gives an example of the convergence of the central deflexion as the iterations increase for a well chosen and a less well chosen damping factor value.

41. In 31-35 Dr Chaplin refers to his approach to the automatic calculation and adjustment of the near-optimal damping factors for the dynamic relaxation method. I have been aware of this work but have never tried to implement it. It appears that both storage requirements and computer run time (depending on how often the damping factors were updated) would increase and may offiset the benefits of enhanced convergence characteristics to some extent.

42. For some years $I$ have been aware that rationalizing the damping factor selection procedure is an aspect of the dynamic relaxation method which merits more attention, particularly for non-linear problems. Otter was also aware of this need at least as long ago as 1968; he had the notion of expressing the damping factors as Chebychev polynomials, which, I believe, would cause the damping to be applied in a manner similar to that outlined by Dr Chaplin in \$ 34. Unfortunately, Otter did not publish his ideas and the matter appears to have attracted scant attention, probably because engineers are able to apply the dynamic relaxation method in the solution of their problems without having to worry unduly about achieving near-optimal damping factors.

\section{References}

22. Chaplin T. K. Metadynamic relaxation applied to automatic analysis of slabs, plates and beams on elastic foundations. The interaction of structure and foundation. Midland Soil Mechanics and Foundation Engineering Society (now Midland Geotechnical Society), Birmingham, 1972, 76-83.

23. OTter J. R. H. Dynamic relaxation compared with other iterative finite difference methods. Nucl. Engng Des., 1966, 3, No. 1, 183-185.

24. Otrer J. R. H. et al. Dynamic relaxation. Proc. Instn Civ. Engrs, 1966, 35, Dec., 633-656.

25. WOOD W. L. Comparison of dynamic relaxation with three other iterative methods. Engineer, Lond., 1967, 224, No. 5835, 683-687.

26. Hodgkins $W . R$. On the relation between dynamic relaxation and semi-iterative matrix methods. Num. Math., 1967, 9, 446-451. 